\title{
Os fatores de risco envolvidos na obesidade no adolescente: uma revisão integrativa
}

\author{
Risk factors involved in adolescent obesity: an integrative review
}

Simone Carvalho Neves (https://orcid.org/0000-0002-9685-761X) ${ }^{1}$

Luciana Miranda Rodrigues (https://orcid.org/0000-0001-8664-9529) ${ }^{2}$

Paulo Alexandre de Souza São Bento (https://orcid.org/0000-0002-1598-3340) ${ }^{3}$

Maria Cecília de Souza Minayo (https://orcid.org/0000-0001-6187-9301) ${ }^{4}$

${ }^{1}$ Enfermagem Souza Marques, Fundação Técnico-Educacional Souza Marques. Av.

Ernani Cardoso 335/345 Cascadura. 21310.310 Rio de Janeiro RJ Brasil. scneves23@gmail.com ${ }^{2}$ Instituto Nacional de

Traumatologia e Ortopedia Jamil Haddad (INTO). Rio de Janeiro RJ Brasil. ${ }^{3}$ Instituto Nacional de Saúde da Mulher, da Criança e do Adolescente Fernandes Figueira (IFF), Fundação Oswaldo Cruz. Rio de Janeiro RJ Brasil. ${ }^{4}$ Departamento de Estudos sobre Violência e Saúde Jorge Careli (Claves), Escola Nacional de Saúde Pública, Fundação Oswaldo Cruz. Rio de Janeiro RJ Brasil.
Abstract The scope of this study was to examine the risk factors for the development of obesity in adolescence. The objective was to identify risk factors for adolescent obesity by means of an Integrative Review of the Literature. The PICO strategy was used to formulate the following central line of inquiry: What are the risk factors for the development of obesity during adolescence? The VHL and EBSCOhost research databases were consulted, resulting in a selection of 25 articles for in-depth study. The phenomenon of obesity was understood as the result of biological, social, psychological, and nutritional factors. The research revealed a lack of consensus on the risks and benefits, which makes evidence-based recommendations difficult. The study identified proposals that can be implemented, such as a change in eating habits, weight control and the practice of physical exercise. Such behavioral changes can be recommended within the context of the family, schools, and health services. The review recommends prevention strategies and the recognition of school as the ideal medium for health promotion through education. From a political and social standpoint, it is necessary to challenge the prevalence of publicity of the food industry that entices adolescents to consume processed foods with high fat and sugar content.

Key words Adolescent, Chronic disease, Health in schools, Obesity
Resumo Os fatores de risco para o desenvolvimento da obesidade na adolescência constituem o objeto deste estudo. Por meio de uma revisão integrativa da literatura, buscou-se identificar os fatores de risco da obesidade nos adolescentes. Utilizou-se a estratégia PICO para se formular a seguinte pergunta norteadora: quais são os fatores de risco para o desenvolvimento da obesidade na adolescência? Os portais de pesquisa consultados foram: BVS e EBSCOhost, com o corpus do estudo ficando com 25 artigos. O fenômeno da obesidade foi entendido como resultante de fatores biológicos, sociais, psicológicos e nutricionais. Constatouse que não existe um consenso sobre riscos e benefícios, o que dificulta recomendações de evidência. Foram identificadas, ainda, propostas passíveis de serem implementadas: a modificação de hábitos alimentares, o controle de peso e a prática de exercícios físicos. Tais modificações comportamentais podem ser recomendadas para os contextos familiares, escolares e dos serviços de saúde. A revisão recomenda estratégias de prevenção e pensar a escola como um espaço rico para promoção da saúde por intermédio da educação. Do ponto de vista político e social, é preciso enfrentar as propagandas da indústria alimentícia, que seduzem os adolescentes a consumirem alimentos processados e ricos em gordura e açucar.

Palavras-chave Adolescente, Doença crônica, Saúde escolar, Obesidade 


\section{Introdução}

A adolescência é uma fase de transição da infância para a vida adulta e se situa entre 10 e 19 anos $^{1}$. No Brasil, de acordo com o Censo Demográfico de 2010, a população de adolescentes era de aproximadamente 18 milhões entre 10 e 14 anos e de 17 milhões entre 15 e 19 anos de idade ${ }^{2}$.

Viero e Farias ${ }^{3}$ ressaltam que essa fase é caracterizada por grandes e múltiplas mudanças, constituindo-se em um período potencialmente difícil, com vários desafios e vulnerabilidades referentes às transformações inerentes ao processo de amadurecimento humano. Uma dessas vulnerabilidades está relacionada ao surgimento da obesidade, que se constitui como um problema de saúde pública. Ela é considerada uma das doenças crônicas não transmissíveis (DNCT) de forte incidência entre os jovens na atualidade, de forma que, se não houver intervenções efetivas para tratá-la, tenderá a se agravar ao longo da vida.

A obesidade é definida como um distúrbio nutricional e metabólico de origem multifatorial, um estado em que o percentual de gordura corporal no indivíduo se encontra elevado por causa de um desequilíbrio entre a ingesta e o gasto de energia. Fatores genéticos, emocionais e estilos de vida estão intimamente relacionados à sua gênese ou manutenção ${ }^{4}$.

A obesidade nos adolescentes pode sobrevir tanto da genética como da ingestão de grande quantidade de gordura e de calorias. Além disso, a falta de atividades físicas e muito tempo despendido nas redes sociais, em jogos interativos e em frente à televisão podem contribuir para o acirramento do problema ${ }^{5}$. Os adolescentes que convivem com a obesidade tendem a se tornar adultos obesos e a apresentar complicações clínicas provenientes do sobrepeso, assim como ter sua expectativa de vida diminuída ${ }^{4}$.

A situação aqui tratada não é exclusiva do Brasil. A prevalência de obesidade e sobrepeso em adolescentes é observada em vários países, como nos Estados Unidos e em nações latino-americanas ${ }^{4}$. Dados da Organização Pan-Americana da Saúde $(\mathrm{OPAS})^{6}$ mencionam que a taxa de obesidade em crianças e adolescentes em todo o mundo foi de 1\% em 1975 (equivalente a 5 milhões de meninas e 6 milhões de meninos), e de 6\% em 2016 (correspondendo a 50 milhões de meninas e quase 74 milhões de meninos). A mesma OPAS ressalta que o número de obesos com idade entre 5 e 19 anos cresceu mais de dez vezes, passando de 11 milhões em 1975 para 124 milhões em $2016^{6}$. Embora os fatores associados ao fenômeno sejam múltiplos, não se pode desconsiderar o peso do marketing das indústrias de alimentos e das políticas que o sustentam. Tampouco o fato de, na maioria dos países, os alimentos mais nutritivos e saudáveis ainda serem muito caros e inacessíveis às famílias e comunidades com baixo poder aquisitivo ${ }^{7}$.

Pela evidência epidemiológica do aumento da obesidade, a OPAS/OMS (Organização Mundial de Saúde) vem recomendando aos países membros que implantem e mantenham sistemas de vigilância sobre os fatores de risco. Em 1989, foi criado no Brasil o Programa Saúde do Adolescente, com múltiplos objetivos, sendo um deles a prevenção das doenças crônicas, entre as quais a obesidade (PROSAD) $)^{4,8}$. Esse programa possibilitou alguns desdobramentos, como a recente realização da Pesquisa Nacional de Saúde do Escolar $(\mathrm{PeNSE})^{9}$, uma parceria estratégica entre o Ministério da Saúde e o Instituto Brasileiro de Geografia e Estatística (IBGE), com apoio do Ministério da Educação e Cultura (MEC). Um de seus objetivos foi identificar prioridades para o desenvolvimento de políticas públicas para a promoção da saúde em adolescentes'. Seu foco foi a vigilância de risco e a proteção contra doenças crônicas. Esse estudo levantou vários hábitos de vida não saudáveis hoje comuns entre jovens, como o consumo de tabaco, álcool e drogas ilícitas, a alimentação inadequada e o sedentarismo.

Diante da importância empírica da obesidade na adolescência e da necessidade de atuar contra ela, este artigo tem como foco realizar uma revisão integrativa da literatura sobre os fatores de risco da obesidade em adolescentes.

\section{Métodos}

Trata-se de um estudo de revisão integrativa de literatura (RIL), utilizando como base teórica os conceitos e métodos propostos por Soares e colaboradores ${ }^{10}$. No campo da saúde, a RIL se baseia na sumarização de achados científicos, no intuito de identificar e compreender problemas, situações e vulnerabilidades relacionadas à população ${ }^{10}$. Exige dos autores o estabelecimento de hipóteses e conclusões sobre o tema em pauta, sendo um trabalho complexo que se alicerça na proposta de colaboração e integração de diversas disciplinas com o objetivo de identificar práticas baseadas em evidências ${ }^{11}$.

Utilizou-se a estratégia $\mathrm{PICO}^{12,13}$ para elaborar a questão norteadora deste estudo, uma vez que ela propicia uma busca acurada das evi- 
dências científicas relacionadas ao objeto. PICO é um acrônimo em que a letra $\mathrm{P}$ (population) indica a população, a letra I (intervention) está relacionada à intervenção, C (comparison) diz respeito à comparação e a letra $\mathrm{O}$ (outcome) se refere aos desfechos esperados. $\mathrm{O}$ estudo comparativo de Methley ${ }^{13}$ sobre vários tipos de revisão sugere que a estratégia PICO permanece sendo a que possui maior sensibilidade para buscas em diferentes bases de dados.

Nesse sentido, para este estudo o acrônimo determinado foi: $\mathrm{P}$ - adolescente, I - obesidade, $\mathrm{C}$ - não se aplica, $\mathrm{O}$ - identificar os fatores de risco para a obesidade. Sendo assim, estabeleceu-se a seguinte pergunta norteadora: quais são os fatores de risco para o desenvolvimento da obesidade na adolescência?

Para o levantamento dos manuscritos, os bancos de dados utilizados foram: Biblioteca Virtual em Saúde (BVS) e EBSCOhost, nas seguintes bases de dados: National Library of Medicine (Medline via PubMed), Literatura Latino-Americana e do Caribe em Ciências da Saúde (LILACS) e Pesquisa Acadêmica Premier (Academic Search Premier - ASP). Utilizaram-se os descritores oficiais (DeCS, 2017): adolescente/ adolescent; doença crônica/chronic disease; saúde escolar/school health; obesidade/obesity.

O cruzamento foi feito pela busca avançada, utilizando-se o booleano and. A busca foi realizada por meio dos descritores adolescente and doença crônica and saúde escolar and obesidade, com suas respectivas terminologias em inglês. Como filtros, artigos nos idiomas português, inglês e espanhol publicados de janeiro de 2007 a dezembro de 2017.

Os critérios de exclusão referentes aos textos encontrados foram: artigos com participantes de pesquisa menores que 10 anos e maiores que 18 anos; artigos sobre adolescentes com doenças transmissíveis; situações de hospitalização e estudos de fármacos ou laboratoriais.

Os artigos elencados para esta RIL foram organizados em um quadro sinóptico, contendo: periódico/revista; título; autores; tipo de abordagem; participantes; cenário da pesquisa; ano de publicação; nível de evidência; local do estudo; e principais resultados. O fluxograma na Figura 1 representa a estruturação do corpus desta revisão.

A análise dos artigos foi pautada no GRADE (Grading of Recommendations Assessment, Development and Evaluation). O GRADE é um sistema desenvolvido por um grupo de pesquisadores com o objetivo de graduar a qualidade das evidências e a força das recomendações. O nível de evidência representa a confiança na informação utilizada em apoio a determinada recomendação, e a avaliação da qualidade da evidência é classificada em quatro níveis: alto, moderado, baixo e muito baixo ${ }^{14}$.

Cabe ressaltar que este tipo de estudo dispensa a apreciação do Comitê de Ética e Pesquisa. Foram utilizados apenas manuscritos de domínio público e todos os critérios éticos referentes à preservação de autoria e citação das fontes foram respeitados.

\section{Resultados e discussão}

A seleção dos artigos foi realizada mediante busca nos bancos de dados Medline ( $\mathrm{n}=106)$, LILACS $(\mathrm{n}=3)$ e ASP $(\mathrm{n}=49)$, chegando-se ao total parcial de 158 artigos. Inicialmente, foram excluídos 63 artigos que não atendiam aos critérios de inclusão (filtros do estudo - Figura 1). Seguindo a análise inicial da busca por evidências, 52 artigos foram excluídos após a leitura dos títulos e dos resumos, por não responderem adequadamente à pergunta PICO. Foram retirados 14 artigos por conta das seguintes situações: duplicata, por estarem inelegíveis, por serem estudos qualitativos, estudos de revisão ou por não estarem disponíveis para leitura. Logo, 25 estudos foram incluídos para leitura na íntegra.

Dos 25 artigos incluídos na revisão integrativa, todos se encontravam na base de dados Medline (Quadro 1). Desse total, 14 foram publicados nos últimos seis anos (2013-2017), e 11 entre 2007-2011. Os artigos selecionados foram publicados em periódicos dos Estados Unidos (20\%), de países da Oceania (20\%), da Europa (16\%), da Índia (12\%), da Austrália (8\%), da América Central (8\%), da China (8\%), do Canadá (4\%) e do Irã (4\%). A maioria dos autores realizou pesquisa seccional $(76 \%)$, os demais utilizaram abordagem longitudinal (12\%), amostragem multiestágio estratificado ( $8 \%$ ) e estudo knockout realizado em quatro momentos (4\%).

Foi feita a leitura minuciosa e crítica de cada artigo. Os trabalhos apresentaram uma multiplicidade de resultados associados aos fatores de risco da obesidade na adolescência. A discussão do presente estudo foi dividida em três categorias, que contemplaram os fatores biológicos ${ }^{15-19}$, os fatores sociais $^{20-37} \mathrm{e}$ os fatores nutricionais ${ }^{38,39}$. A proposta foi realizar uma discussão não dicotômica do fenômeno, ou seja, os achados desta revisão revelaram que analisar a obesidade exige um olhar que considere a multiplicidade dessa condição. 

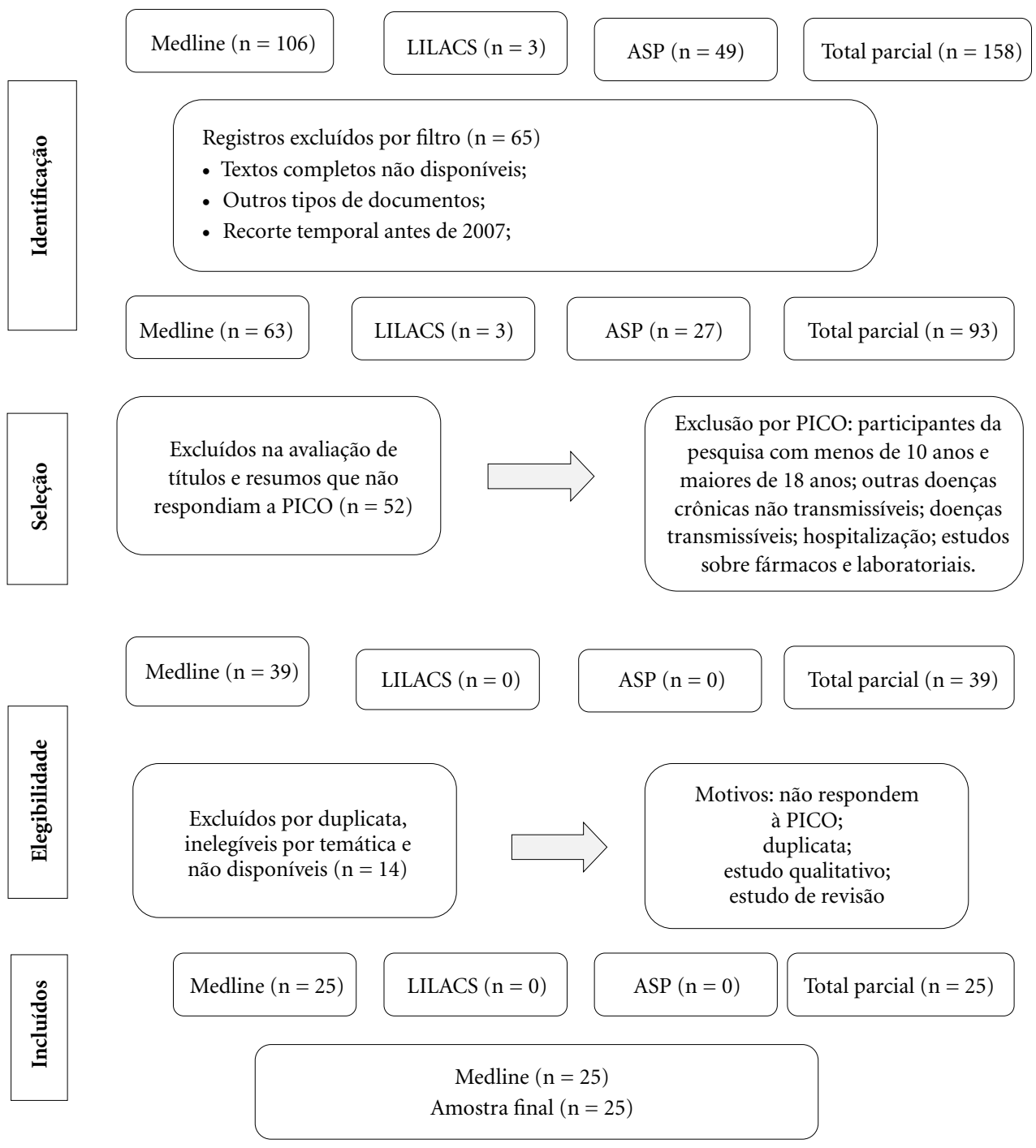

Figura 1. Fluxograma das etapas realizadas na seleção dos artigos.

Fonte: Elaborado pelos autores.

\section{Aspectos biomédicos da obesidade na adolescência}

Coutinho $^{40}$ enfatiza que o "mapa gênico da obesidade humana" continua se desenvolvendo rapidamente a cada ano, à medida que mais genes e regiões cromossômicas são relacionados a ela. Esse mapa gênico identificou mais de 430 genes associados a fenótipos de obesidade humana $^{41}$. No entanto, Clement e Ferre ${ }^{42}$ relatam que inúmeros marcadores genéticos já foram associados com a obesidade e suas consequências metabólicas, mas as interações específicas entre genótipo e fenótipo nas formas poligênicas de obesidade permanecem mal compreendidas.

Por sua vez, o campo da epigenética traz contribuições para o entendimento da multifatorialidade encontrada nesta revisão. Os autores asseveram que existem evidências de que a exposição a diversas condições ambientais em etapas iniciais da vida pode induzir alterações persistentes no epigenoma. Contudo, as evidências também sugerem que algumas marcas epigenéticas são modificáveis por hábitos de vida relacionados à alimentação e à atividade física. Essas evidências podem contribuir para a prevenção da obesidade 
Quadro 1. Sinopse dos dados coletados nos artigos da revisão integrativa de 2007 a 2017 organizados por autores, ano de publicação, local do estudo, título, objetivos e tipo de estudo.

\begin{tabular}{|c|c|c|c|c|}
\hline $\begin{array}{l}\text { Número } \\
\text { do } \\
\text { artigo }\end{array}$ & $\begin{array}{c}\text { Autor, ano de } \\
\text { publicação e } \\
\text { local de estudo }\end{array}$ & Título & Objetivos & $\begin{array}{c}\text { Delineamento } \\
\text { do estudo }\end{array}$ \\
\hline A1 & $\begin{array}{l}\text { Merlo et al. }{ }^{38} \\
(2015) \\
\text { EUA }\end{array}$ & $\begin{array}{l}\text { Práticas escolares para aumentar a } \\
\text { disponibilidade de frutas, vegetais e } \\
\text { grãos e reduzir o sódio na refeição } \\
\text { na escola - Estados Unidos da } \\
\text { América (EUA), 2000, 2006 e } 2014 \text {. }\end{array}$ & $\begin{array}{l}\text { Examinar a prevalência de práticas, } \\
\text { relacionadas com a implementação das } \\
\text { normas nutricionais na escola. }\end{array}$ & $\begin{array}{l}\text { Estudo } \\
\text { seccional }\end{array}$ \\
\hline A2 & $\begin{array}{l}\text { Song et al. }{ }^{20} \\
(2013) \\
\text { EUA }\end{array}$ & $\begin{array}{l}\text { Tendência secular da prevalência } \\
\text { da obesidade em crianças na China } \\
\text { entre } 1985 \text { e 2010: disparidade de } \\
\text { gênero. }\end{array}$ & $\begin{array}{l}\text { Identificar se a diferença de gênero } \\
\text { quanto à obesidade tornou-se maior ou } \\
\text { menor nos últimos } 25 \text { anos em crianças } \\
\text { chinesas em idade escolar, ou seja, se } \\
\text { o ritmo de aumento foi diferente para } \\
\text { meninos e meninas. }\end{array}$ & $\begin{array}{l}\text { Estudo } \\
\text { seccional }\end{array}$ \\
\hline A3 & $\begin{array}{l}\text { Mbowe et al. }{ }^{30} \\
(2014) \\
\text { Guatemala }\end{array}$ & $\begin{array}{l}\text { Prevalência da síndrome } \\
\text { metabólica e os fatores de risco } \\
\text { associados em crianças de uma } \\
\text { escola na Guatemala. }\end{array}$ & $\begin{array}{l}\text { Investigar a prevalência da síndrome } \\
\text { metabólica e fatores de risco } \\
\text { cardiovasculares associados à síndrome } \\
\text { metabólica entre escolares de uma } \\
\text { população predominantemente de } \\
\text { ascendência maia passando por uma } \\
\text { rápida urbanização. }\end{array}$ & $\begin{array}{l}\text { Estudo } \\
\text { seccional }\end{array}$ \\
\hline A4 & $\begin{array}{l}\text { Martinez, } \\
\text { Ruelas, } \\
\text { Granger }^{18} \\
(2017) \\
\text { EUA } \\
\end{array}$ & $\begin{array}{l}\text { Associação entre índice de massa } \\
\text { corporal e ácido úrico salivar } \\
\text { entre crianças, jovens e adultos de } \\
\text { origem mexicana: diferenças de } \\
\text { desenvolvimento e gênero. }\end{array}$ & $\begin{array}{l}\text { Explorar como os níveis de ácido úrico } \\
\text { na saliva diferem entre crianças de } \\
\text { origem mexicana em estágios distintos } \\
\text { de desenvolvimento. }\end{array}$ & $\begin{array}{l}\text { Estudo } \\
\text { seccional }\end{array}$ \\
\hline A5 & $\begin{array}{l}\text { Ichiho, Robles, } \\
\text { Aitaoto }^{23} \\
(2013) \\
\text { Norte da } \\
\text { Islândia } \\
\end{array}$ & $\begin{array}{l}\text { Uma avaliação de doenças não } \\
\text { transmissíveis, diabetes e fatores de } \\
\text { risco relacionados na comunidade } \\
\text { das Ilhas Marianas do Norte: uma } \\
\text { perspectiva sistêmica. }\end{array}$ & $\begin{array}{l}\text { Avaliar a capacidade dos sistemas } \\
\text { administrativo, clínico, de suporte e de } \\
\text { dados para abordar os problemas das } \\
\text { DCNT, incluindo diabetes e seus fatores } \\
\text { de risco. }\end{array}$ & $\begin{array}{l}\text { Estudo } \\
\text { seccional }\end{array}$ \\
\hline A6 & $\begin{array}{l}\text { Ichiho et } \\
\text { al. }^{28}(2013) \\
\text { Oceania }\end{array}$ & $\begin{array}{l}\text { Uma avaliação de doenças não } \\
\text { transmissíveis, diabetes e fatores } \\
\text { de risco relacionados no território } \\
\text { da Samoa Americana: uma } \\
\text { perspectiva sistêmica. }\end{array}$ & $\begin{array}{l}\text { Descrever a carga de DCNT, avaliar a } \\
\text { capacidade e as atividades relacionadas } \\
\text { a prestação de serviços, coleta de dados } \\
\text { e relatórios identificando os problemas. }\end{array}$ & $\begin{array}{l}\text { Estudo } \\
\text { seccional }\end{array}$ \\
\hline A7 & $\begin{array}{l}\text { Ichiho et al }{ }^{25} \\
(2013) \\
\text { Ilhas Marshall }\end{array}$ & $\begin{array}{l}\text { Uma avaliação de doenças não } \\
\text { transmissíveis, diabetes e fatores } \\
\text { de risco relacionados na República } \\
\text { das Ilhas Marshall, Majuro Atoll: } \\
\text { uma perspectiva sistêmica. }\end{array}$ & $\begin{array}{l}\text { Avaliar a capacidade dos sistemas } \\
\text { administrativo, clínico, de suporte e de } \\
\text { dados para abordar os problemas das } \\
\text { DCNT, incluindo diabetes e seus fatores } \\
\text { de risco. }\end{array}$ & $\begin{array}{l}\text { Estudo } \\
\text { seccional }\end{array}$ \\
\hline A8 & $\begin{array}{l}\text { Ichiho et al } \\
29 .(2013) \\
\text { Palau }\end{array}$ & $\begin{array}{l}\text { Uma avaliação de doenças não } \\
\text { transmissíveis, diabetes e fatores de } \\
\text { risco relacionados na República de } \\
\text { Palau: uma perspectiva sistêmica. }\end{array}$ & $\begin{array}{l}\text { Avaliar a capacidade dos sistemas } \\
\text { administrativo, clínico, de suporte e de } \\
\text { dados para abordar os problemas das } \\
\text { DCNT, incluindo diabetes e seus fatores } \\
\text { de risco. }\end{array}$ & $\begin{array}{l}\text { Estudo } \\
\text { seccional }\end{array}$ \\
\hline
\end{tabular}

em sujeitos ou populações com perfil epigenético desfavorável, no sentido de que o fenômeno não pode ser analisado de forma fragmentada, e sim como resultante de fatores entrelaçados da biologia e das condições culturais ${ }^{43}$.
Na mesma linha, é importante pontuar que os autores que focaram seus estudos nas questões biológicas mencionaram também outros fatores de risco para a obesidade na adolescência. Assim, Lin e colaboradores ${ }^{15}$ fazem considerações sobre 
Quadro 1. Sinopse dos dados coletados nos artigos da revisão integrativa de 2007 a 2017 organizados por autores, ano de publicação, local do estudo, título, objetivos e tipo de estudo.

\begin{tabular}{|c|c|c|c|c|}
\hline $\begin{array}{l}\text { Número } \\
\text { do } \\
\text { artigo }\end{array}$ & $\begin{array}{l}\text { Autor, ano de } \\
\text { publicação e } \\
\text { local de estudo }\end{array}$ & Título & Objetivos & $\begin{array}{c}\text { Delineamento } \\
\text { do estudo }\end{array}$ \\
\hline A9 & $\begin{array}{l}\text { Ichiho et al. }{ }^{26} \\
(2013) \\
\text { Micronesia }\end{array}$ & $\begin{array}{l}\text { Avaliação de doenças não } \\
\text { transmissíveis, diabetes e fatores } \\
\text { de risco relacionados nos estados } \\
\text { federados da Micronésia, Estado } \\
\text { de Pohnpei: uma perspectiva } \\
\text { sistêmica. }\end{array}$ & $\begin{array}{l}\text { Avaliar a capacidade dos sistemas } \\
\text { administrativo, clínico, de suporte e de } \\
\text { dados para abordar os problemas das } \\
\text { DCNT. }\end{array}$ & $\begin{array}{l}\text { Estudo } \\
\text { seccional }\end{array}$ \\
\hline A10 & $\begin{array}{l}\text { Ichiho et al. }{ }^{27} \\
(2013) \\
\text { República } \\
\text { Marshall }\end{array}$ & $\begin{array}{l}\text { Uma avaliação de doenças não } \\
\text { transmissíveis, diabetes e fatores } \\
\text { de risco relacionados na República } \\
\text { do Atol Kwajelein da Ilha Marshall, } \\
\text { Ilha Ebeyve: uma perspectiva } \\
\text { sistêmica. }\end{array}$ & $\begin{array}{l}\text { Avaliar a capacidade dos sistemas } \\
\text { administrativo, clínico, de suporte e de } \\
\text { dados para abordar os problemas das } \\
\text { DCNT, incluindo diabetes e seus fatores } \\
\text { de risco. }\end{array}$ & $\begin{array}{l}\text { Estudo } \\
\text { seccional }\end{array}$ \\
\hline A11 & $\begin{array}{l}\text { Singh, Yu, } \\
\text { Kogan }^{22} \\
(2013) \\
\text { EUA }\end{array}$ & $\begin{array}{l}\text { Saúde, condições crônicas } \\
\text { e disparidades de risco } \\
\text { comportamental entre crianças e } \\
\text { adolescentes imigrantes dos EUA. }\end{array}$ & $\begin{array}{l}\text { Examinar as diferentes prevalências } \\
\text { de } 23 \text { países quanto a saúde, condição } \\
\text { crônica e indicadores comportamentais } \\
\text { entre } 91.532 \text { crianças filhas de } \\
\text { imigrantes e de crianças americanas. }\end{array}$ & $\begin{array}{l}\text { Estudo } \\
\text { seccional }\end{array}$ \\
\hline A12 & $\begin{array}{l}\text { Lin et al. }{ }^{15} \\
(2015) \\
\text { Estocolmo, } \\
\text { Atenas, } \\
\text { Heraklion, } \\
\text { Roma, Zaragoza }\end{array}$ & $\begin{array}{l}\text { Ingestão de proteína animal } \\
\text { e vegetal e suas associações } \\
\text { com obesidade e indicadores } \\
\text { cardiometabólicos em adolescentes } \\
\text { europeus: o estudo transversal } \\
\text { HELENA. }\end{array}$ & $\begin{array}{l}\text { Avaliar a ingestão de proteína } \\
\text { animal e de vegetais em adolescentes } \\
\text { europeus estratificados por gênero e } \\
\text { idade e investigando suas associações } \\
\text { com indicadores cardiometabólicos } \\
\text { (antropometria e biomarcadores). }\end{array}$ & $\begin{array}{l}\text { Estudo } \\
\text { seccional }\end{array}$ \\
\hline A13 & $\begin{array}{l}\text { Patel et al. }{ }^{32} \\
(2017) \\
\text { Índia }\end{array}$ & $\begin{array}{l}\text { Conformidade de doenças crônicas } \\
\text { dentro dos lares indianos: um } \\
\text { estudo transversal. }\end{array}$ & $\begin{array}{l}\text { Estimar a associação entre viver com } \\
\text { alguém com uma condição crônica e o } \\
\text { próprio estado de condição crônica. }\end{array}$ & $\begin{array}{l}\text { Estudo } \\
\text { seccional }\end{array}$ \\
\hline A14 & $\begin{array}{l}\text { Passmore et al. } \\
37 \text { (2017) } \\
\text { Austrália }\end{array}$ & $\begin{array}{l}\text { O impacto de um programa } \\
\text { liderado pela comunidade que } \\
\text { promove perda de peso e vida } \\
\text { saudável em comunidades } \\
\text { aborígenes: Knockout Health } \\
\text { Challenge em Nova Gales do Sul. }\end{array}$ & $\begin{array}{l}\text { Identificar programas eficazes de estilo } \\
\text { de vida saudável para abordar fatores } \\
\text { de risco para doenças crônicas entre os } \\
\text { povos aborígenes. }\end{array}$ & \begin{tabular}{|l|} 
Estudo \\
Knockout \\
Health \\
Challenge \\
(realizado \\
em quatro \\
momentos) \\
\end{tabular} \\
\hline A15 & $\begin{array}{l}\text { Ekta, Tulika }{ }^{21} \\
(2016) \\
\text { Índia }\end{array}$ & $\begin{array}{l}\text { Distribuição dos fatores de risco } \\
\text { para doenças cardiovasculares } \\
\text { entre meninos e meninas do } \\
\text { ensino médio na área urbana de } \\
\text { Dibrugarh Assam. }\end{array}$ & $\begin{array}{l}\text { Comparar a distribuição dos fatores } \\
\text { de risco entre meninos e meninas de } \\
\text { Assam. }\end{array}$ & $\begin{array}{l}\text { Estudo } \\
\text { seccional }\end{array}$ \\
\hline A16 & $\begin{array}{l}\text { Leatherdale }^{19} \\
(2015) \\
\text { Canadá }\end{array}$ & $\begin{array}{l}\text { Exame de ocorrência dos fatores de } \\
\text { risco modificáveis associados com } \\
\text { doença crônica entre jovens no } \\
\text { estudo COMPASS. }\end{array}$ & $\begin{array}{l}\text { Examinar a prevalência de fatores de } \\
\text { risco para o câncer e como os fatores } \\
\text { demográficos e sociais estão associados } \\
\text { à ocorrência de fatores de risco entre os } \\
\text { jovens no estudo COMPASS. }\end{array}$ & $\begin{array}{l}\text { Estudo } \\
\text { longitudinal }\end{array}$ \\
\hline
\end{tabular}

o excessivo consumo de proteína animal e vegetal por jovens obesos. A maioria dos adolescentes por eles estudados encontrava-se no estágio de Tanner 3 ou 4, quando a presença da proteína é funda- mental para a o crescimento ósseo e muscular. No entanto, o excesso de ingesta adicional de proteína torna-se um fator de risco para o desenvolvimento de doenças crônicas, entre elas a obesidade. 
Quadro 1. Sinopse dos dados coletados nos artigos da revisão integrativa de 2007 a 2017 organizados por autores, ano de publicação, local do estudo, título, objetivos e tipo de estudo.

\begin{tabular}{|c|c|c|c|c|}
\hline $\begin{array}{l}\text { Número } \\
\text { do } \\
\text { artigo }\end{array}$ & $\begin{array}{c}\text { Autor, ano de } \\
\text { publicação e } \\
\text { local de estudo }\end{array}$ & Título & Objetivos & $\begin{array}{c}\text { Delineamento } \\
\text { do estudo }\end{array}$ \\
\hline A17 & $\begin{array}{l}\text { Guavamzadeh, } \\
\text { Khalkhali, } \\
\text { Alizadeh }^{31} \\
(2013) \\
\text { Irã }\end{array}$ & $\begin{array}{l}\text { Visualização de TV, } \\
\text { independentemente da atividade } \\
\text { física e de alimentos obesogênicos } \\
\text { aumenta sobrepeso e obesidade em } \\
\text { adolescentes. }\end{array}$ & $\begin{array}{l}\text { Estimar a prevalência de sobrepeso e } \\
\text { obesidade e fatores de risco associados } \\
\text { em uma amostra representativa de } \\
\text { estudantes de } 11 \text { a } 20 \text { anos. }\end{array}$ & $\begin{array}{l}\text { Estudo } \\
\text { seccional }\end{array}$ \\
\hline A18 & $\begin{array}{l}\text { Crinall et al. }{ }^{35} \\
(2017) \\
\text { Austrália }\end{array}$ & $\begin{array}{l}\text { Risco de doença cardiovascular em } \\
\text { jovens australianos indígenas: uma } \\
\text { foto atual dos cuidados preventivos } \\
\text { de saúde. }\end{array}$ & $\begin{array}{l}\text { Examinar a assistência preventiva à } \\
\text { saúde e o registro de diabetes tipo } \\
2 \text { e fatores de risco para doenças } \\
\text { cardiovasculares e seu manejo em } \\
\text { jovens aborígenes e ilhéus do Estreito } \\
\text { de Torres (indígenas australianos) em } \\
\text { centros de atenção primária à saúde } \\
\text { (PHCs). }\end{array}$ & $\begin{array}{l}\text { Estudo de } \\
\text { coorte }\end{array}$ \\
\hline A19 & $\begin{array}{l}\text { Verstraeten et } \\
\text { al. }^{33} \\
(2016) \\
\text { Equador }\end{array}$ & $\begin{array}{l}\text { Fatores individuais e ambientais } \\
\text { influenciando o comportamento } \\
\text { alimentar dos adolescentes de } \\
\text { baixa e média renda. }\end{array}$ & $\begin{array}{l}\text { Testar a validade de uma estrutura } \\
\text { conceitual. }\end{array}$ & $\begin{array}{l}\text { Estudo } \\
\text { seccional }\end{array}$ \\
\hline A20 & $\begin{array}{l}\text { Ricci-Cabello } \\
\text { et al. } .^{36} \\
(2015) \\
\text { Inglaterra }\end{array}$ & $\begin{array}{l}\text { Impacto da prevalência de } \\
\text { condições concordantes e } \\
\text { discordantes na qualidade dos } \\
\text { cuidados de diabetes em práticas } \\
\text { familiares na Inglaterra }\end{array}$ & $\begin{array}{l}\text { Examinar a associação entre a } \\
\text { prevalência de diabetes-concordante } \\
\text { e diabetes-condições discordantes } \\
\text { e a qualidade do tratamento desse } \\
\text { problema no nível da prática familiar na } \\
\text { Inglaterra. }\end{array}$ & $\begin{array}{l}\text { Estudo } \\
\text { seccional }\end{array}$ \\
\hline A21 & $\begin{array}{l}\text { Dong et al. }{ }^{16} \\
(2015) \\
\text { China }\end{array}$ & $\begin{array}{l}\text { Associações entre indicadores } \\
\text { de adiposidade e pressão } \\
\text { arterial elevada entre crianças e } \\
\text { adolescentes chineses. }\end{array}$ & $\begin{array}{l}\text { Avaliar a prevalência do aumento da } \\
\text { pressão arterial usando sexo-idade e seu } \\
\text { risco atribuível por conta do sobrepeso } \\
\text { e da obesidade em crianças chinesas de } \\
7 \text { a } 17 \text { anos. }\end{array}$ & $\begin{array}{l}\text { Estudo de } \\
\text { amostragem } \\
\text { multiestágio } \\
\text { estratificado }\end{array}$ \\
\hline A22 & $\begin{array}{l}\text { Ortega et al. }{ }^{24} \\
(2013) \\
\text { Itália, Suíça, } \\
\text { Estônica e } \\
\text { Espanha }\end{array}$ & $\begin{array}{l}\text { Atividade física objetivamente } \\
\text { mensurada e tempo sedentário } \\
\text { durante a infância, adolescência e } \\
\text { juventude: um estudo de coorte. }\end{array}$ & $\begin{array}{l}\text { Estudar mudanças em medidas objetivas } \\
\text { (acelerometria) de atividade física } \\
\text { moderada a vigorosa e tempo sedentário } \\
\text { desde a infância até a adolescência e da } \\
\text { adolescência até a idade adulta jovem. }\end{array}$ & $\begin{array}{l}\text { Estudo } \\
\text { de coorte } \\
\text { longitudinal }\end{array}$ \\
\hline A23 & $\begin{array}{l}\text { Gulati et al. }{ }^{34} \\
(2013) \\
\text { Índia }\end{array}$ & $\begin{array}{l}\text { Ingestões dietéticas e correlatos } \\
\text { familiares de sobrepeso/obesidade: } \\
\text { um estudo de quatro cidades na } \\
\text { Índia. }\end{array}$ & $\begin{array}{l}\text { Avaliar conhecimento, atitude e } \\
\text { prática de nutrição, atividade física e } \\
\text { outros estilos de vida em uma amostra } \\
\text { nacionalmente representativa de } \\
\text { crianças e mães urbanas na Índia. }\end{array}$ & $\begin{array}{l}\text { Estudo } \\
\text { seccional }\end{array}$ \\
\hline A24 & $\begin{array}{l}\text { Nianogo et al. }{ }^{39} \\
(2016) \\
\text { EUA }\end{array}$ & $\begin{array}{l}\text { Associações entre autopercepção } \\
\text { de peso, intenções de escolha } \\
\text { de alimentos e resposta do } \\
\text { consumidor à informação calórica: } \\
\text { uma investigação retrospectiva } \\
\text { de clientes de centros de saúde } \\
\text { pública no condado de Los Angeles } \\
\text { antes da implementação da } \\
\text { regulamentação de rotulagem nos } \\
\text { cardápios. }\end{array}$ & $\begin{array}{l}\text { Examinar associações de autopercepção } \\
\text { de peso (medidas pelo peso corporal) } \\
\text { com intenções de escolha alimentar e } \\
\text { resposta do consumidor à informação } \\
\text { calórica entre adultos de baixa renda } \\
\text { durante a era do regulamento de } \\
\text { rotulagem de cardápio. }\end{array}$ & $\begin{array}{l}\text { Estudo } \\
\text { seccional }\end{array}$ \\
\hline A25 & $\begin{array}{l}\text { Dong et al. }{ }^{17} \\
(2015) \\
\text { China }\end{array}$ & $\begin{array}{l}\text { Risco de pressão alta em crianças } \\
\text { chinesas com sobrepeso e } \\
\text { obesidade. }\end{array}$ & $\begin{array}{l}\text { Estimar a contribuição do sobrepeso e } \\
\text { da obesidade associados à pressão alta } \\
\text { em crianças chinesas. }\end{array}$ & $\begin{array}{l}\text { Estudo de } \\
\text { amostragem } \\
\text { multiestágio } \\
\text { estratificado }\end{array}$ \\
\hline
\end{tabular}


Umas das consequências do aumento do peso corporal é a alteração da pressão arterial. Esse mecanismo ocorre porque o adipócito é uma célula que atualmente tem sido associada à produção de vários mediadores que podem participar como mecanismos fisiopatológicos da hipertensão arterial sistêmica (HAS) associada à obesidade $^{44}$.

Os estudos de Dong e colaboradores ${ }^{16,17}$ associaram a obesidade como fator de risco na prevalência de HAS. O índice de massa corporal (IMC) foi considerado o melhor parâmetro para calcular sobrepeso/obesidade na avaliação de pressão arterial alterada entre crianças e adolescentes. O IMC também foi utilizado no estudo de Martinez, Ruelas e Granger ${ }^{18}$, mostrando que jovens obesos com IMC aumentado apresentam taxas de ácido úrico alteradas e que isso é um indício de DCNT. No Brasil, o acompanhamento da criança e do adolescente é pautado pelo gráfico de estatura versus idade e IMC versus idade, ambos presentes na Caderneta do Adolescente ${ }^{45}$.

Leatherdale ${ }^{19}$ analisou a prevalência de fatores de risco modificáveis que são associados ao câncer. Ressaltou em seu estudo que, do total de adolescentes analisados, $6,2 \%$ apresentaram obesidade, $13,8 \%$ estavam com sobrepeso, $53,1 \%$ foram classificados como inativos fisicamente, 96,7\% como altamente sedentários e $95,1 \%$ não se alimentavam de forma saudável. $\mathrm{O}$ autor associou tais hábitos ao risco de câncer no futuro. $\mathrm{O}$ controle do peso, os hábitos e atitudes de vida saudável são importantes para a prevenção da obesidade e, consequentemente, de outras DCNT.

Crianças e adolescentes com obesidade têm maior risco de desenvolver doenças crônicas como cardiopatias, acidente vascular encefálico, HAS, dislipidemias, diabetes melito, aterosclerose, entre outras ${ }^{46}$. Essas doenças são diagnosticadas em adultos, mas atualmente têm sido cada vez mais diagnosticadas em crianças e adolescentes.

Sobre os dados apresentados nessa primeira categoria, cabe o alerta da $\mathrm{OMS}^{47}$, que, sem desconsiderá-los, pede cautela na análise antropométrica dos adolescentes, tendo em vista as importantes mudanças corporais e hormonais por que passam, sendo difícil estabelecer um diagnóstico definitivo de obesidade nessa etapa da vida.

\section{Fatores sociais envolvidos na obesidade de adolescentes}

Essa categoria remete aos problemas de saúde relacionados às condições em que uma pessoa vive e trabalha. Ou seja, diz respeito aos fatores sociais, econômicos, culturais, étnicos/raciais, de gênero e psicológicos ${ }^{48}$.

Song et al. ${ }^{20} \mathrm{e}$ Ekta et al. ${ }^{21}$ identificaram que a prevalência da obesidade é maior nos adolescentes do sexo masculino. A disparidade entre os sexos está associada à visão sobre a imagem corporal masculina - no caso citado pelo autor, do homem oriental - para quem a obesidade não é prejudicial à saúde. Mas sabe-se que, em geral, a diferença na prevalência da obesidade nos adolescentes dos sexos masculino e feminino pode ser influenciada pela etnia, podendo ser justificada pelo cromossomo sexual ou pelos efeitos organizacionais dos hormônios gonadais ${ }^{49}$.

O estudo de Singh, Yu e Kogan ${ }^{22}$ apontou que os comportamentos de risco dos adolescentes imigrantes nos EUA variam de acordo com sua etnia, cultura e o tempo de imigração. Os que apresentam níveis mais altos de sedentarismo são mais suscetíveis do que os nativos. Os autores assinalam, no entanto, que a cada geração observase uma diminuição da taxa de inatividade física entre os adolescentes imigrantes.

Ichiho, Robles e Aitaoto $^{23}$ relataram que as principais causas de mortalidade por doenças cardíacas, derrame e câncer estão associadas às altas taxas de sobrepeso e obesidade. No grupo etário de 15 anos, o sobrepeso e a obesidade cresceram drasticamente. Houve um aumento de horas dispensadas pelos adolescentes para assistir TV e, por conseguinte, uma diminuição da frequência nas aulas de educação física.

O estudo de Ortega e colaboradores ${ }^{24}$ corro- $^{-}$ bora os resultados de Ichiho, Robles e Aitaoto ${ }^{23}$ ao evidenciar que a atividade física de moderada a vigorosa diminui da infância para a adolescência. O sedentarismo tende a aumentar nessa transição, assim como o risco de desenvolver obesidade e outras doenças crônicas. Assim, o incentivo da escola e dos familiares é importante para que os adolescentes passem a ter interesse pela prática de exercícios físicos, fazendo que elas sejam prazerosas e agreguem valores para a saúde.

Os estudos de Ichiho e colaboradores ${ }^{25-29}$ realizados em países da Oceania (Samoa Americana, República de Marshall, Palau e Micronésia) mostram que, lá como aqui, os fatores de risco associados ao sobrepeso e à obesidade abrangem hábitos alimentares não saudáveis, sedentarismo e adoecimento e mortes por DCNT.

Os estudos de Mbowe e colaboradores ${ }^{30}$ e de Ghavamzadeh, Khalkhali e Alizade ${ }^{31}$ incluem o universo escolar. Os autores ressaltam que o nível educacional das mães, o tipo de escola e o tem- 
po despendido assistindo TV estão associados ao aumento de sobrepeso e obesidade, assim como a ingesta de alimentos obesogênicos e a falta de atividades físicas.

Uma situação que causou estranhamento em nossa análise é o fato de as taxas de obesidade diminuírem em até $32 \%$ quando havia uma criança adicional no agregado familiar, em estudo realizado na Guatemala, onde o índice de desenvolvimento humano (IDH) era de 0,616 em 2013 e onde há indícios de insegurança alimentar no âmbito familiar ${ }^{50}$. Essa situação certamente se relaciona às condições socioeconômicas dos jovens e de suas famílias.

Patel et al..$^{32}$, Verstraeten et al. ${ }^{33} \mathrm{e}$ Gulati et al. ${ }^{34}$ afirmam que a obesidade nos pais se reflete na obesidade dos filhos. O comportamento alimentar dos adolescentes está inserido em uma interação complexa com seu meio cultural, e os hábitos alimentares constituem uma rotina de longa duração. Os fatores ambientais estão relacionados à permissividade parental na escolha e no gosto pelos alimentos por parte dos adolescentes.

Um dos problemas tratados por Crinall e colaboradores ${ }^{35}$ é a obesidade e a situação clínica de jovens indígenas na Austrália quanto ao diabetes tipo 2. Os autores elencam os fatores de risco para as DCNT, entre elas a obesidade, e chamam atenção para as intervenções na cultura que provocam doenças e para a importância de ações preventivas direcionadas à vida social e à saúde.

$\mathrm{O}$ atendimento dos serviços de saúde deve ser pautado pelo acolhimento do usuário com vistas à resolutividade. No mesmo sentido apontado por Crinallet al. ${ }^{35}$, Ricci-Cabello e colaboradores $^{36}$ chamam atenção para que se dê atenção ao atendimento dos adolescentes com DCNT, incluindo a obesidade. A pessoa com comorbidade normalmente frequenta mais o serviço de saúde e tem mais chances de que suas necessidades sejam atendidas. Esses serviços devem estar preparados para ofertar um atendimento acolhedor e eficaz.

O estudo de Passmore e colaboradores ${ }^{37}$ foi realizado com aborígenes (índios australianos) por meio de uma metodologia que os autores consideraram exitosa, intitulada "Desafio". Eles desenvolveram um método que leva à perda de peso e incentiva a atividade física e uma boa nutrição. O conjunto de ações contribuiu para que os adolescentes melhorassem suas condições de saúde e reduzissem a obesidade e o risco de outras DCNT.

No Brasil, há o Programa Nacional de Atenção à Saúde do Povo Indígena ${ }^{50}$. Entretanto, existem poucas informações sobre a relação entre saúde e nutrição nesse grupo, sobretudo entre os adolescentes. Essa lacuna dificulta a avaliação dos casos de obesidade nesse segmento populacional. Castro e colaboradores ${ }^{51}$, por exemplo, compararam os resultados de seus estudos com adolescentes brasileiros não indígenas com levantamentos feitos com indígenas. E concluíram que as medidas antropométricas associadas ao sobrepeso e à obesidade nesse último grupo são semelhantes ou superiores, mostrando a importância do acompanhamento dos jovens indígenas. Sá $(2018)^{52}$ chama atenção para vários agravos que estão ocorrendo com esse grupo, em especial doenças endócrinas, nutricionais e metabólicas, frequentemente decorrentes da obesidade.

\section{Aspectos nutricionais da obesidade na adolescência}

Nesta categoria foram analisados dois artigos. Um abordou questões sobre o consumo de alimentos ricos em calorias e sódio, pobres em nutrientes e os ultra processados. Esse estudo foi realizado em escolas incluídas no Programa Nacional de Refeição Escolar, por meio de um levantamento periódico feito pelo Centro de Controle de Doenças (CDC) dos Estados Unidos. O outro artigo estudou a autopercepção dos adolescentes quanto ao peso e os rótulos de alimentos que são consumidos. Esse estudo foi conduzido pelo Departamento de Saúde Pública de Los Angeles (EUA) com adolescentes e adultos de baixa renda.

Merlo et al. ${ }^{38}$ realizaram um estudo para examinar as escolas em relação às práticas determinadas pelas normas nutricionais. Foram analisados os dados coletados por meio de uma pesquisa nacional realizada pelo CDC em 2000, 2006 e 2014 , a fim de verificar a qualidade dos alimentos ofertados aos adolescentes, tais como: frutas, legumes, grãos integrais e sódio. Os resultados mostraram, no decorrer de dez anos, a maior oferta de alimentos com baixo teor de sódio, a substituição do sal por outros temperos, o aumento do consumo de vegetais, grãos integrais e a maior oferta de frutas. No entanto, a maioria dos adolescentes dos EUA não adere às recomendações nacionais para uma dieta saudável, com o risco de ganho de peso, obesidade, diabetes e outras doenças. Aproximadamente $90 \%$ das crianças e adolescentes dos EUA consomem mais sódio do que o recomendado.

Esse estudo chama atenção para à iniciativa do governo brasileiro, que em 2009 regulamentou o Programa Nacional de Alimentação Escolar (PNAE) por meio da Lei $n^{\circ} 11.947^{53}$, criada para 
assegurar que as cantinas das escolas públicas ofertem refeições saudáveis que atendam às necessidades nutricionais dos alunos, educando-os para que obtenham hábitos benéficos à saúde. A iniciativa brasileira quanto ao consumo de alimentos saudáveis, quando pensada a partir do estudo de Merlo et al. ${ }^{38}$, é vanguardista, pois foi promulgada seis anos antes do artigo. Uma proposição do governo no investimento em promoção da saúde no ambiente escolar.

Olhando-se do ponto de vista mais micro, a escola é um cenário com possibilidades para se trabalhar assuntos de interesse coletivo dentro da perspectiva educacional. Por isso, os Parâmetros Curriculares Nacionais (PCN) do Brasil propõem que temas transversais, como a saúde, sejam contemplados com a mesma relevância das áreas convencionais de ensino ${ }^{54}$. Até aqui, o eixo avaliado levou em consideração o consumo de alimentos saudáveis sob a perspectiva institucional. O estudo seguinte iluminou a perspectiva dos indivíduos.

Nianogo e colaboradores ${ }^{39}$ empreenderam $^{2}$ um estudo com o objetivo de analisar as associações de autopercepção de peso corporal com as intenções de escolha alimentar e as respostas dos consumidores às informações calóricas. Os resultados mostraram que a autopercepção de peso não parece estar associada ao hábito de ler os rótulos dos alimentos para obter informações acerca das calorias. Para os adolescentes, o conhecimento sobre a composição nutricional dos alimentos é importante para que seja possível fazer escolhas visando a saúde.

Em ambos os estudos os autores se preocuparam com situações macro e micro referentes à alimentação e à obesidade, abordando a qualidade dos alimentos, as estratégias governamentais e a escolha dos indivíduos no consumo de alimentos. Assim, para se pensar em ações preventivas, acesso, qualidade de atendimento, hábitos de vida, escolhas, entre outros aspectos, é importante refletir acerca das condições que levam os indivíduos à obesidade. A questão geoeconômica é, do ponto de vista global, um fator limitante de acesso ao alimento. Por situação de pobreza e miséria, as pessoas socialmente mais vulneráveis optam por comprar e consumir produtos baratos e, por conseguinte, com altos teores de gordura, açúcar e $\mathrm{sal}^{55}$, o que resulta em aumento da obesidade com subnutrição ou desnutrição no mundo.

Nas pesquisas aqui revistas, fica claro que os padrões dietéticos dos adolescentes são influenciados pela indústria de alimentos, com as propagandas publicitárias estimulando o consumo de alimentos processados ricos em gordura e açúcar, o que tem um apelo especial entre famílias e comunidades pobres.

A análise dos estudos permite uma reflexão sobre o contexto das mudanças que mais impactam a vida dos adolescentes, que se encontram em transformações físicas, psicológicas e sociais, marcadas de forma particular pela progressiva emancipação da família e da escola.

Os trabalhos permitiram responder à questão de pesquisa a respeito dos fatores de risco que impactam o desenvolvimento da obesidade na adolescência: do ponto de vista global, a questão geoeconômica das empresas de processamento de alimentos; a alteração da pressão arterial; a maior prevalência em indivíduos do sexo masculino em culturas onde a massa corporal de homens é entendida como marcador de saúde; o sedentarismo aumentado em imigrantes; o aumento de horas dispensadas pelos adolescentes diante das telas da TV, do computador e nas redes sociais, em detrimento às possibilidades de atividades físicas; a diminuição da frequência nas aulas de educação física; o nível educacional das mães; o reflexo de pais obesos na obesidade dos filhos; o consumo em excesso de alimentos ricos em calorias e sódio mas pobre em nutrientes, bem como os ultraprocessados; e a autopercepção do peso. Esses fatores de risco também elevam as chances para o desenvolvimento de outras DCNT.

A análise foi baseada no GRADE, para graduar a qualidade das evidências e a força das recomendações. Observa-se que, dada à variabilidade de resultados dos estudos analisados e dos locais onde as pesquisas empíricas foram feitas, a pesquisa não permite evidências precisas ${ }^{14}$. Entretanto, certa generalização do conhecimento sobre os fatores de risco é possível e ajuda a formular algumas propostas de ação, como: ajudar os jovens a criarem hábitos de alimentação balanceada a partir da escola, ensinando-lhes a evitar alimentos ultraprocessados; incentivar a prática de exercícios físicos; e orientar quanto ao risco do uso excessivo do computador, da TV e das redes sociais.

A situação da obesidade é complexa e multifatorial, sendo necessário o acompanhamento das famílias e dos adolescentes por meio do estreitamento da relação entre a escola e a Unidade Básica de Saúde, para o desenvolvimento de ações de saúde por intermédio do atendimento multiprofissional da demanda reprimida, das visitas domiciliares e de programas de reeducação alimentar. 


\section{Conclusão}

A obesidade na adolescência é um problema de saúde pública que afeta não apenas o crescimento e o desenvolvimento físico. Interfere também em questões sociais, emocionais e em vivências de situações estigmatizantes. O estudo mostrou que a obesidade na adolescência está associada a fatores pré-existentes que colaboram para o surgimento do aumento de peso, sendo eles principalmente biológicos, sociais e nutricionais. Em geral, cada um deles tem um impacto diferenciado de acordo com as circunstâncias, mas atuam de forma inter-relacionada e complexa. Daí a dificuldade de isolar apenas um fator associado.
Uma das limitações desta revisão é a impossibilidade de definir os fatores preponderantes, dada a variabilidade geográfica, cultural e de foco dos temas analisados. Isso dificulta a elaboração de recomendações possíveis para o problema levantado. No entanto, tomando como base os fatores de risco que foram levantados nos estudos, é possível dizer que os jovens podem ser protagonistas na transformação de sua situação e, frequentemente, de sua família, na medida em que tomem conhecimento das questões implicadas na obesidade e se tornem multiplicadores desse conhecimento. Nesse sentido, a escola e as Unidades Básicas de Saúde têm um papel fundamental e insubstituível.

\section{Colaboradores}

Todos os autores participaram da elaboração do artigo, leram e aprovaram a versão final. SC Neves participou da confecção e delineamento do estudo, da coleta de dados, da execução do procedimento metodológico, da análise e interpretação dos dados e da redação do manuscrito; LM Rodrigues e PAS São-Bento contribuíram na confecção e delineamento do estudo, na coleta de dados, na execução do procedimento metodológico, na análise e interpretação dos dados e na redação do manuscrito. MCS Minayo participou da interpretação dos dados, da análise crítica, da revisão e aprovação da versão final. 


\section{Referências}

1. Brasil. Ministério da Saúde (MS). A saúde de adolescentes e jovens: uma metodologia de auto-aprendizagem para equipes de atenção básica de saúde. Brasília: MS; 2012.

2. Brasil. Instituto Brasileiro de Geografia e Estatística (IBGE). Sinopse do Censo Demográfico 2010. Rio de Janeiro: IBGE; 2011. [acessado 2017 Maio 8]. Disponível em: http://www.ibge.gov.br/home/estatistica/ populacao/censo2010/sinopse/default_sinopse.shtm

3. Viero VSF, Farias JM. Educational actions for awareness of a healthier lifestyle in adolescents. J Phys Educ 2017; 28:e2812.

4. Brasil. Ministério da Saúde (MS). Secretaria de Vigilância em Saúde. Departamento de Análise de Situação de Saúde. Plano de ações estratégicas para a saúde do adolescente. Brasília: MS; 2017.

5. FUNDAÇ̃̃O ABRINQ. Cenário da infância e adolescência no Brasil 2018. [acessado 2019 Julho 2] Disponível em: https://observatorio3setor.org.br/ wp-content/uploads/2018/04/cenario_da_infancia_2018_internet.pdf

6. Organização Pan-Americana da Saúde Brasil (OPAS). Obesidade entre crianças e adolescentes aumentou dez vezes em quatro décadas, revela novo estudo do Imperial College London e da OMS; 2017. [acessado 2018 Abril 4]. Disponível em: http://www.paho.org/bra/ index.php?option $=$ com_content $\&$ view $=$ article\&i$\mathrm{d}=5527$ :obesidade-entre-criancas-e-adolescentes-aumentou-dez-vezes-emquatro-decadas-revela-novo -estudo-do-imperial-college-london-e-da-oms\&Ite$\operatorname{mid}=820$

7. Organização Pan-Americana da Saúde (OPAS). Cuidados inovadores para condições crônicas: organização e prestação de atenção de alta qualidade às doenças crônicas não transmissiveis nas Américas. Washington, DC OPAS; 2015. [acessado em 2018 Abril 4]. Disponível em: https://www.paho.org/hq/dmdocuments/2015/ent -cuidados-innovadores-InnovateCCC-digital-PT.pdf

8. Jager ME, Batista FA, Perrone CM, Santos SS, Dias ACG. O adolescente no contexto da saúde pública brasileira:reflexões sobre o PROSAD. Psicol Estud 2014; 19(2):211-221.

9. Oliveira MM, Campos MO, Andreazzi MAR, Malta DC. Características da Pesquisa Nacional de Saúde do Escolar - PeNSE. Epidemiol Serv Saude 2017; 26(3):605-616

10. Soares CB, Hoga LAK, Peduzzi M, Sangaleti C, Yonekura T, Silva D. Revisão integrativa: conceitos e métodos utilizados na enfermagem. Rev Esc Enferm USP 2014; 48(2):335-345.

11. Oliveira WA, Silva JL, Sampaio JMC, Silva MAI. Saúde do escolar: uma revisão integrativa sobre família e bullying. Cien Saude Colet 2017; 22(5):1553-1564.

12. Santos CMC, Pimenta CAM, Nobre MRC. A estratégia PICO para a construção da pergunta de pesquisa e busca de evidências. Rev Latino-am Enferm 2007; 15(3):508-511.

13. Methley AM, Campbell S, Chew-Graham C, McNally R, Cheraghi-Sohi S. PICO, PICOS and SPIDER: a comparison study of specificity and sensitivity in three search tools for qualitative systematic reviews. BMC Health Serv Res 2014; 14:579.
14. Brasil. Ministério da Saúde (MS). Secretaria de Ciência, Tecnologia e Insumos Estratégicos. Departamento de Ciência e Tecnologia. Diretrizes metodológicas: Sistema GRADE - Manual de graduação da qualidade da evidência e força de recomendação para tomada de decisão em saúde. Brasília: MS; 2014.

15. LinY, Mouratidou T, Vereecken C, Kersting M, Bolca S, Moraes ACF, Cuenca-García M, Moreno LA, González-Gross M, Valtueña J, Labayen I, Grammatikaki E, Hallstrom L, Leclercq C, Ferrari M, Gottrand F, Beghin L, Manios Y, Ottevaere1 C, Van Oyen H, Molnar D, Kafatos A, Widhalm K, Gómez-Martinez S, Prieto LED, De Henauw S, Huybrechts I. Nutrition Journal 2015, 14(1):10-21.

16. Dong B, Wang Z, Wang HJ, Ma J. Population attributable risk of overweight and obesity for high blood pressure in Chinese children. Blood Press 2015; 24(1):230-36.

17. Dong B,Wang Z, Wang HJ. Ma J. Associations between adiposity indicators and elevated blood pressure among Chinese children and adolescents. J Hum Hypertens 2015; 29:236-240.

18. Martínez AD, Ruelas L, Granger DA. Association between body mass index and salivary uric acid among Mexican-origin infants, youth and adults: gender and developmental differences. Dev Psychobiol 2017; 59(2):225-234

19. Leatherdale ST. An examination of the co-occurrence of modifiable risk factors associated with chronic disease among youth in the COMPASS study. Cancer causes control 2015; 26(4):519-528.

20. Song Y, Wang HJ, Ma J, Wang Z. Secular trends of obesity prevalence in urban chinese children from 1985 to 2010: gender disparity. PLoS One 2013; 8(1):e53069.

21. Ekta G, Tulika MG. Risk factor distribution for cardiovascular diseases among high school boys and girls of urban Dibrugarh, Assam. J Family Med Prim Care 2016; 5(1):108-113.

22. Singh GK, Yu SM, Kogan, MD. Health, chronic conditions, and behavioral risk disparities among U.S. immigrant children and adolescents. Public Health Rep 2013; 128(1):463-479.

23. Ichiho HM, Robles B, Aitaoto N. An assessment of non-communicable diseases, diabetes, and related risk factors in the Commonwealth of the Northern Mariana Islands: a systems perspective. Hawaii J Med Public Health 2013; 72(5 Suppl. 1):19-29.

24. Ortega FB, Konstabel K, Pasquali E, Ruiz JR, Hurtig-Wennlo A, Maestu J, Lof M, Harro J, Bellocco R, Labayen I, Veidebaum T, Sjostrom M. Objectively measured physical activity and sedentary time during childhood, adolescence and young adulthood: a cohort study. PLoS One 2013; 8(4):e60871.

25. Ichiho HM, DeBrum I, Kedi S, Langidrik J, Aitaoto N. An assessment of non-communicable diseases, diabetes, and related risk factors in the Republic of the Marshall Islands, Majuro Atoll: a systems perspective. Hawaii J Med Public Health 2013; 72(5 Suppl. 1):8797. 
26. Ichiho HM, Anson R; Keller E, Lippwe K, Aitaoto N An assessment of non-communicable diseases, diabetes, and related risk factors in the federated states of Micronesia, State of Pohnpei: a systems perspective. Hawaii J Med Public Health 2013; 72 (5 Suppl. 1):4956.

27. Ichiho HM, Seremai J, Trinidad R, Paul I, Langidrik J, Aitaoto N. An assessment of non-communicable diseases, diabetes and related risk factors in the Republic of the Marshall Islands, Kwajelein Atoll, Ebeye Island: a systems perspective. Hawaii J Med Public Health 2013; 72 (5 Suppl. 1):77-86.

28. Ichiho HM, Roby FT, Ponausuia ES, Aitaoto N. An assessment of non-communicable diseases, diabetes, and related risk factors in the territory of American Samoa: a systems perspective. Hawaii J Med Public Health 2013; 72 (5 Suppl. 1):10-18.

29. Ichiho HM, Demei Y, Kuartei S, Aitaoto N. An assessment of non-communicable diseases, diabetes, and related risk factors in the republic of Palau: a systems perspective. Hawaii J Med Public Health 2013; 72(5 Suppl. 1):98-105.

30. Mbowe O, Diaz A, Wallace J, Mazariegos M, Jolly P. Prevalence of metabolic syndrome and associated cardiovascular risk factors in Guatemalan school children. Matern Child Health J 2014; 18(7):1619-1627.

31. Ghavamzadeh S, Khalkhali HR, Alizadeh M. TV viewing, independent of physical activity and obesogenic foods, increases overweight and obesity in adolescents. J Health Popul Nutr 2013; 31(3):334-342.

32. Patel SA, Dhillon PK, Kondal D, Jeemon P, Kahol K, Manimunda SP, Purty AJ, Deshpande A, Negi PC, Ladhani S, Toteja GS, Patel V, Prabhakaran D. Chronic disease concordance within Indian households: a cross-sectional study. PLoS Med 2017; 14(9):e1002395.

33. Verstraeten R, Leroy JL, Pieniak Z, Ochoa-Avilès A, Holdsworth M, Verbeke W, Maes L, Kolsteren P. Individual and environmental factors influencing adolescents' dietary behavior in low- and middle-income settings. PLoS One 2016; 11(7):e0157744.

34. Gulati S, Misra A, Colles SL, Kondal D, Gupta N, Goel K, Bansal S, Mishra M, Madkaikar V, Bhardwaj S. Dietary intakes and familial correlates of overweight/ obesity: a four-cities study in India. Ann nutr metab 2013; 62(4):279-290.

35. Crinall B, Boyle J, Gibson-Helm M, Esler D, Larkins S, Bailie R. Cardiovascular disease risk in young Indigenous Australians: a snapshot of current preventive health care. Aust N Z J Public Health 2017; 41(5):460466.

36. Ricci-Cabello I, Stevens S, Kontopantelis E, Dalton ARH, Griffiths RI, Campbell JL, Doran T, Valderas JM. Impact of the prevalence of concordant and discordant conditions on the quality of diabetes care in family practices in England. Ann Fam Med 2015; 13(6):514-522.

37. Passmore E, Shepherd B, Milat A, Maher L, Hennessey K, Havrlant R, Maxwel M, Hodge W, Christian F, Richards J, Mitchel J. The impact of a community-led program promoting weight loss and healthy living in Aboriginal communities: the New South Wales Knockout Health Challenge. BMC Public Health 2017; 17(1):951-960.
38. Merlo C, Brener N, Kann L, McManus T, Harris D Mugavero K. School-level practices to increase availability of fruits, vegetables, and whole grains, and reduce sodium in school meals - United States, 2000, 2006, and 2014. MMWR Morb Mortal Wkly Rep 2015; 64(33):905-908.

39. Nianogo RA, Kuo T, Smith LV, Arah OA. Associations between self-perception of weight, food choice intentions, and consumer response to calorie information a retrospective investigation of public health center clients in Los Angeles County before the implementation of menu labeling regulation. BMC Public Health 2016; 16:60-69.

40. Coutinho W. Etiologia da obesidade [informativo]. Ano VII - n. 30, maio de 2007.

41. Snyder EE, Walts B, Perusse L, Chagnon IC, Weisnagel SJ, Rankinen T, Bouchard C. The human obesity gene map: the 2003 update. Obes Res 2004; 12(3):369-439.

42. Clément K, Ferré P. Genetics and the pathophysiology of obesity. Pediatr Res 2003; 53(5):721-725.

43. Casanello P, Krause BJ, Castro-Rodríguez JA, Uauy R. Epigenética y obesidad. Rev Chil Pediatr 2016; 87(5):335-342.

44. Barroso SG, Abreu VG, Francischetti EA. A participação do tecido adiposo visceral na gênese da hipertensão e doença cardiovascular aterogênica. Um conceito emergente. Arq Bras Cardiol 2002; 78(6):618-630.

45. Brasil. Ministério da Saúde (MS). Caderneta do adolescente. 2ed. 1a reimp. Brasília: MS, 2012. [acessado em 2019 Ago 23]. Disponível em: http://www.saude. gov.br/saude-para-voce/saude-do-adolescente-e-dojovem/caderneta-do-adolescente

46. Turke KC, Saraiva DJB, Lantieri CJB, Ferreira JFM, Chagas ACP. Fatores de risco cardiovascular: o diagnóstico e prevenção devem iniciar nas crianças e adolescentes. Rev Soc Cardiol Estado de São Paulo 2019; 29(1):25-27.

47. World Health Organization (WHO). Physical Status: the use and interpretation of antropometry [report]; 1995. [acessado em 2019 Jan 14]. Disponível em: https://apps.who.int/iris/bitstream/handle/10665 /37003/WHO_TRS_854.pdf;jsessionid=9DF7E 67E5 DC036C3AC65F98B89D8081E?sequence $=1$

48. World Health Organization (WHO). World conference on social determinants of health [report]; 2011. [acessado em 2019 Jan 14]. Disponível em: https://www. who.int/sdhconference/resources/Conference_Report.pdf

49. Wisniewski AB, Chernausek SD. Gender in childhood obesity: family environment, hormones, and genes. Gend Med 2009; 6(1):76-85.

50. Organização Pan-americana de Saúde (OPAS). Desigualdade exacerba fome, desnutrição e obesidade na América Latina e no Caribe [editorial]. [acessado em 2019 Apr 02]. Disponível em: https://www.paho.org/ bra/index.php?option $=$ com_content\&view $=$ article\&id=5799:desigualdade-exacerba-fome-desnutricao-e-obesidade-na-america-latina-e-no-caribe\&Itemid $=839$

51. Castro TG, Barufaldi LA, Schlüssel MM, Conde WL, Leite MS, Schuch I. Waist circumference and waist circumference to height ratios of Kaingáng indigenous adolescents from the State of Rio Grande do Sul, Brazil. Cad Saude Publica 2012; 28(11):2053-2062. 
52. Sá RAR. Avaliação do risco de doenças cardiovasculares em indígenas Krenak do estado de Minas Gerais [dissertação]. Belo Horizonte: Universidade Federal de Minas Gerais; 2018.

53. Brasil. Lei no 11.947 , de 16 de junho de 2009. Dispõe sobre o atendimento da alimentação escolar e do Programa Dinheiro Direto na Escola aos alunos da educação básica. Diário Oficial da União 2009; 16 jun.

54. Copetti J, Folmer V. Educação e saúde no contexto escolar. Uruguaiana: Universidade Federal do Pampa; 2015.

55. Organização Pan-Americana de Saúde (OPAS). Obesidade entre crianças e adolescentes aumentou dez vezes em quatro décadas, revela novo estudo do Imperial College London e da OMS [editorial]. [acessado em 2019 Apr 20]. Disponível em: https://www.paho.org/bra/ index.php?option $=$ com_content $\&$ view $=$ article\&i$\mathrm{d}=5527$ :obesidade-entre-criancas-e-adolescentes-aumentou-dez-vezes-em-quatro-decadas-revela-novo -estudo-do-imperial-college-london-e-da-oms\&Itemid $=820$

Artigo apresentado em 19/10/2019

Aprovado em 02/03/2020

Versão final apresentada em 04/03/2020

Editores-chefes: Romeu Gomes, Antônio Augusto Moura da Silva 